\title{
Assessment of residual gastric volume and thirst in patients who drink before gastroscopy
}

\author{
S M Greenfield, G J M Webster, A S Brar, K Ah Mun, E R Beck, F R Vicary
}

\begin{abstract}
Background-Before endoscopy patients undergo an uncomfortable fluid fast to reduce the risk of gastric acid aspiration and to ensure good endoscopic views are obtained. However, fluids rapidly leave the stomach and thus a long fluid fast before endoscopy may not be required. Aims-The object of this study was to establish whether drinking before endoscopy is safe and relieves patients' symptoms of thirst.

Patients and Methods-88 patients with American Society of Anaesthesiologists classification of physical status grades I and II were recruited in a controlled randomised single blind trial. The volume and pH of gastric aspirate obtained at gastroscopy was assessed in patients who drank 330 $\mathrm{ml}$ of water a minimum 90 minutes before their endoscopy and compared with values in patients who starved overnight.
\end{abstract}

Results-44 patients who drank ('drinkers') $330 \mathrm{ml}$ of water a mean 117 minutes before their morning gastroscopy had a similar volume and $\mathrm{pH}$ of gastric aspirate compared with 44 patients starved overnight ('starvers'); median volume $12.5 \mathrm{ml}$ versus $10 \mathrm{ml}$, median $\mathrm{pH}$ $2 \cdot 0$ versus $2 \cdot 0$; 'drinkers' versus 'starvers' (NS). Before endoscopy patients were asked to score their thirst and hunger ratings as either none, mild, moderate or severe. Ratings for moderate and severe thirst were grouped together for analysis. The percentage of drinkers compared with starvers in each group with no thirst, mild thirst, and moderate severe thirst was $63 \%, 46 \%$, and $37 \%$ respectively ( $\chi^{2}$ test for trend $p<0 \cdot 05)$. Hunger ratings were similar in the two groups.

Conclusions-It is safe for elective day cases to drink a significant volume of water two hours before endoscopy and this alleviates symptoms of thirst.

(Gut 1996; 39: 360-362)

Keywords: endoscopy, endoscopy practice, endoscopy safety, fluids, drinking.

The risks and consequences of gastric acid aspiration during anaesthesia are reduced by minimising the gastric volume and increasing the $\mathrm{pH}$ of gastric contents. ${ }^{1-3}$ Therefore, patients are deprived of food and fluid from midnight prior to a morning anaesthetic and only permitted a light breakfast before an afternoon anaesthetic. A similar protocol is followed for patients undergoing upper gastrointestinal endoscopy to prevent acid aspiration. However, this prolonged deprivation of fluid is not in keeping with the physiology of gastric emptying as clear fluids rapidly leave the stomach. ${ }^{4}$ Thus the conventional fast before endoscopy may be unnecessary and reducing the fasting time for fluid is unlikely to increase the risk of pulmonary aspiration and may prove more acceptable to patients. Recently it has been shown that drinking clear fluids up to two hours before a general anaesthetic does not change gastric volumes compared with a standard fast when these are measured by aspiration using a nasogastric (NG) tube. ${ }^{5}{ }^{6} \mathrm{NG}$ tube aspiration is notoriously unreliable, however, and we therefore decided to study whether it is safe for patients to drink before endoscopy and use the gastroscope to accurately aspirate and assess the gastric juice.

The aim of the study was to analyse gastric volumes and $\mathrm{pH}$ in those patients who drank $330 \mathrm{ml}$ of water a minimum 90 minutes before a morning endoscopy and compare these results with patients starved conventionally that is, overnight. We also determined whether endoscopy reduces thirst.

\section{Methods}

Patients requiring endoscopy were recruited from the Gastroenterology and Surgical outpatients at the Whittington Hospital. Patients were excluded for the following reasons: age $<18$ years, inability to understand informed written consent, dysphagia, gastric outlet obstruction suspected, pregnancy, American Society of Anaesthesiologists classification of physical status grade III or greater, previous gastric or duodenal surgery, insulin dependent diabetes $>10$ years or any other suggestion of autonomic neuropathy. Patients taking the following drugs within 14 days of endoscopy were also excluded: mebeverine, hyoscine, metoclopromide, cisapride, erythromycin, opiates, $\mathrm{H}_{2}$ receptor antagonists or proton pump inhibitors.

Randomisation was performed by a staff nurse in clinic and the referring doctor and endoscopist were unaware of the study group to which the patients were allocated. Patients drew an envelope that randomised them to either (a) an overnight fast of food and fluids from midnight ('starvers') or (b) to drinking a $330 \mathrm{ml}$ bottle of supermarket still mineral water (supplied by clinic) at 730 am on the morning of endoscopy ('drinkers'), but no food from midnight. Patients were given written instructions to follow.

Patients were gastroscoped starting at 9 am and no more than three study patients were on 
one list to ensure that a significant time period had not elapsed between the morning drink and the endoscopy in those patients who were so randomised. Prior to endoscopy all patients were asked to score their thirst and hunger rating as either none, mild, moderate or severe as previously described. ${ }^{5} 6$ Patients in the drinking group were also asked to confirm that they drank the entire bottle of water and the time of this drink, while starvers confirmed that they had no morning drink.

Before the gastroscopy sterile water was aspirated through the suction channel of the endoscope and then drained dry. All endoscopies were performed by two doctors (SG and GW). Patients were sedated with midazolam only and pulse oximetry was used throughout the procedure. After oesophageal intubation the endoscopist first performed a complete examination of the stomach and aspirated all the gastric juice present into a sterile container attached to the suction channel of the endoscope before proceeding with the rest of the examination. The volume of gastric juice was measured and an aliquot stored at $-70^{\circ} \mathrm{C}$ for $\mathrm{pH}$ measurement at a later date. After thawing, the gastric juice $\mathrm{pH}$ was measured as described by one of the authors previously using a Corning $\mathrm{pH} 103$ electrode. ${ }^{7}$

We calculated that a sample size of 40 patients in each group was required to achieve a power of $90 \%$ to detect any difference at the $5 \%$ level.

\section{Ethics}

Ethical permission for this study was granted by the ethical committee of the Whittington Hospital NHS Trust.

\section{Statistics}

The Mann-Whitney U test was used to determine any difference between starvers and drinkers in terms of volume and $\mathrm{pH}$ of gastric juice. The $\chi^{2}$ test for trend was used to find out if thirst and hunger was affected by drinking. The Spearman $r$ test was used to test for any correlation between the time from the drink to endoscopy (defined as the drink to endoscopy interval) and the residual gastric volume. The $\mathrm{Z}$ test was used to determine whether the size of the residual gastric volume was related to the presence or absence of duodenal pathology. Significance was taken at the $5 \%$ level.

\section{Results}

A total of 89 patients were recruited. One patient randomised to drinking failed to comply with the instructions and is not included in the final analysis. Patients were well matched for age and sex (Table). All remaining drinkers drank the entire bottle of water and all starvers confirmed that they had fasted from midnight. There were no complications after endoscopy.

For drinkers the mean time from their drink to endoscopy was 117 minutes (95\% CI of mean 110 to 124 minutes). This figure is
Demographic data and median (range) volume and $\mathrm{pH}$ of gastric aspirate. There was no significant difference between starvers and drinkers in the volume and $p H$ of gastric aspirate. $\mathrm{pH}$ analysis was performed on the samples of 39 drinkers and 36 starvers as in some cases there was no gastric juice aspirate

\begin{tabular}{lll}
\hline & Drinkers & Starvers \\
\hline Number & 44 & 44 \\
M/F & $24 / 20$ & $24 / 20$ \\
Age (y) (mean (SD)) & $46 \cdot 1(14 \cdot 4)$ & $48 \cdot 5(15 \cdot 7)$ \\
Gastric volume (ml) & $12 \cdot 5(0-81)$ & $10(0-72)$ \\
pH & $2 \cdot 0(1 \cdot 62-7 \cdot 4)$ & $2 \cdot 0(1 \cdot 62-7 \cdot 14)$ \\
\hline
\end{tabular}

greater than the originally anticipated 90 minutes as patients drank their water up to half an hour earlier than requested and also up to three patients were studied on each dedicated endoscopy list. Results in the Table show that there was no significant difference between starvers and drinkers in the volume and $\mathrm{pH}$ of gastric aspirate. There was no correlation between the drink to endoscopy interval and the residual gastric volume (RGV) aspirated. For instance, the median volume of the 14 patients who drank their water 90 to 100 minutes before endoscopy was $13 \mathrm{ml}$ (range 2 to $45 \mathrm{ml}$ ). In fact a major determinant of the $\mathrm{RGV}$ was the presence of duodenal pathology at endoscopy; of the 10 patients (starvers $n=4$, drinkers $n=6$ ) in whom a $R G V$ of $31 \mathrm{ml}$ or more was obtained eight of $10(80 \%)$ had duodenal pathology as follows: duodenal ulcer disease $(n=4)$, duodenal ulcer scarring $(n=2)$, deformed duodenum compatible with previous ulceration $(n=1)$, severe duodenitis $(n=1)$, and normal $(n=2)$. This compares with a much lower incidence of duodenal pathology (10 of $78,12 \cdot 8 \%$ ) in the remaining 78 patients in whom a RGV of $30 \mathrm{ml}$ or less was obtained $(p<0.001)$ : duodenal ulceration $(n=3)$, duodenal ulcer scarring $(n=1)$, deformed duodenum $(n=2)$, duodenitis/duodenal erosions $(\mathrm{n}=4)$. However, $\mathrm{pH}$ values were similar in patients with and without duodenal pathology.

The Figure illustrates thirst scores in patients. Moderate and severe scores have been

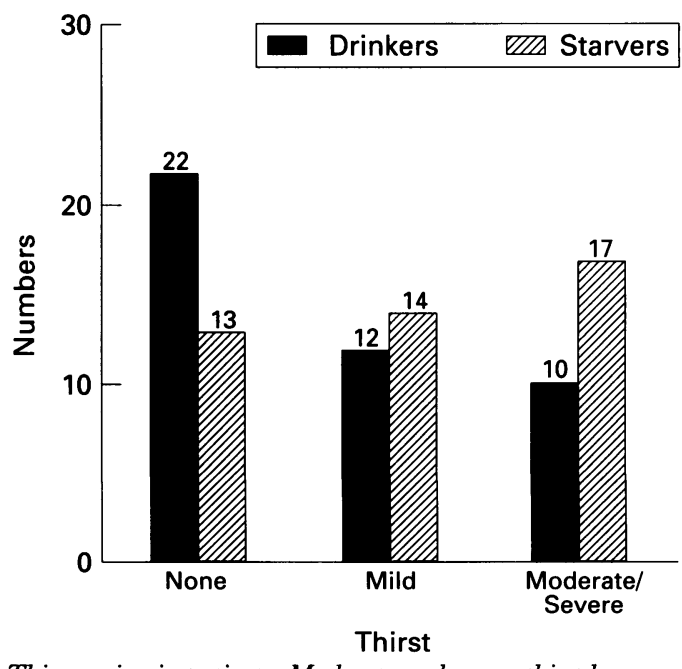

Thirst rating in patients. Moderate and severe thirst has been amalgamated for statistical analysis. The percentage of drinkers compared with starvers in each group was $63 \%$ (no thirst), $46 \%$ (mild thirst), and $37 \%$ (moderate/severe thirst), $\chi^{2}$ test for trend $p<0 \cdot 05$. 
amalgamated for statistical analysis. Drinkers suffered less thirst and the proportion of drinkers to starvers in each group fell as the severity of thirst increased ( $\chi^{2}$ test for trend $\mathrm{p}<0.05)$. However starvers and drinkers had similar hunger ratings; the proportion of drinkers with none, mild or moderate/severe hunger was $51 \%, 47 \%$, and $52 \%$ respectively.

\section{Discussion}

During endoscopy patients are unable to protect their airway adequately as the endoscope affects glottic closure and this puts them at risk of acid aspiration into the lungs. The severity of acid aspiration has previously been thought to be related to the $\mathrm{pH}$ and volume of gastric contents; it has been estimated following extrapolation from animal studies that if the RGV is less than $25 \mathrm{ml}$ and the $\mathrm{pH}>2.5$ then pulmonary acid aspiration is less severe when it occurs. ${ }^{23}$ As a consequence it is standard practice for patients to be starved from midnight before a morning endoscopy. This prolonged fast of fluid is illogical, however, for the following reasons; firstly, the stomach can secrete up to $50 \mathrm{ml} / \mathrm{h}$ of acidic fluid even in fasting patients and secondly, it has been known for some time that fluids leave the stomach rapidly. ${ }^{4}$ Recently anaesthetists have shown that there is no difference in the RGV of patients allowed to drink clear fluids two hours before their anaesthetic compared with those who are not. ${ }^{5}$ In those studies, however, RGV was measured by NG aspiration, which both sets of authors admit may be unreliable. To our knowledge this study is the first in which the RGV of patients who have drunk before sedation has been measured by direct visualisation - that is, during gastroscopy.

Our results confirm that the volume and $\mathrm{pH}$ of gastric juice from patients are not changed by drinking $330 \mathrm{ml}$ of water a mean 117 minutes before endoscopy. Indeed, it is interesting that duodenal pathology seems to be associated with a relatively high $R G V$ although this is perhaps not surprising as patients with duodenal ulcers are known to have a higher gastric acid output compared with normal controls. Drinking before endoscopy did not affect views of the gastric mucosa and there were no respiratory or other complications in the drinking group. Thus if the risk of aspiration is related to the $\mathrm{RGV}$ it is not changed by drinking two hours before endoscopy. Indeed, it is noteworthy that a recent audit of 14149 gastroscopies has shown that respiratory complications after endoscopy may be associated with the use of topical anaesthetic spray on the pharynx before the examination, a procedure not used in our study. ${ }^{9}$ Drinking water before endoscopy resulted in significantly less thirst compared with patients starved conventionally. Although gastroscopy is a relatively rapid procedure causing minimum discomfort to most patients, it is probable that a significant number of patients are anxious about undergoing this examination. This stress will drive sympathetic output, exacerbating thirst in patients who are already feeling dry and thus increase anxiety. Allowing patients to drink before this procedure alleviates symptoms of thirst and keeps patients comfortable. Accordingly, we have changed our instructions sheet at the Whittington Hospital allowing outpatients to drink clear fluids a minimum 120 minutes before endoscopy and we would recommend that they fulfil the exclusion criteria mentioned in this study.

In addition, drinking before endoscopy will allow patients to take their regular oral medication thus controlling conditions, such as angina, that might interfere with this examination. Furthermore, our findings can be extrapolated to patients undergoing colonoscopy who often feel dehydrated having consumed large doses of laxatives. This study also shows that fit patients undergoing elective surgery need not be deprived of clear fluids for more than two hours before anaesthesia, thus maintaining comfort. Certainly the Canadian Anaesthetists' Society have recently published guidelines that a fluid fast of more than three hours is unnecessary in healthy patients undergoing surgery. ${ }^{10}$

Joseph Lister stated in 1883 'While it is desirable that there is no solid matter in the stomach when chloroform is administered, it will be found very salutary to give a cup of tea or beef-tea about two hours previously'. ${ }^{11}$ One hundred and thirteen years later we would recommend similar advice especially with regard to fit patients undergoing elective endoscopy.

We thank the patients who took part in this study and acknowledge the assistance of the outpatient and endoscopy nurses. We thank Mr R Lock for the referral of his patients and the Department of Biochemistry for their help with the $\mathrm{pH}$ measurements.

This work was presented in part to the Spring Meeting of the BSG, Manchester 1995 and appears as an abstract in Gut 1995; 36: A38.

1 Mendelson CL. The aspiration of stomach contents into the lungs during obstetric anesthesia. Am $\mathcal{f}$ Obstet Gynecol 1946; 52: 191-205.

2 Roberts JB, Shirley MA. Reducing the risk of acid aspiration during cesarian section. Anesth Analg 1974; 53: 859-68.

3 James CF, Modell JH, Gibbs CP, Kuck EJ, Ruiz BC. Pulmonary aspiration - effects of volume and $\mathrm{pH}$ in the rat. Anesth Analg 1984; 63: 665-8.

4 rat. Anesth Analg 1984; 63: 665-8. human stomach. F Physiol 1951; 113: 157-68.

Phillips S, Hutchinson S, Davidson T. Preoperative Phillips S, Hutchinson S, Davidson T. Preoperative
drinking does not affect gastric contents. Br $\mathcal{f}$ Anaesth drinking does

6 Hutchinson A, Maltby JR, Reid CRG. Gastric fluid volume and $\mathrm{pH}$ in elective inpatients. Part I: coffee or orange juice versus overnight fast. Can $\mathcal{F}$ Anaesth 1988; 35: 12-5.

7 Powell JJ, Greenfield SM, Thompson RPH. Concentration of metals in human gastric juice in health and peptic ulcer disease. Gut 1992; 33: 1617-20.

8 Guyton AC. Textbook of medical physiology, 7th ed. Philadelphia: WB Saunders 1986; 774-8.

9 Quine MA, Bell GD, McCoy RF, Charlton JE, Devlin HB, Hopkins A. Prospective audit of upper gastrointestinal endoscopy in two regions of England: safety, staffing and endoscopy in two regions of England: sa

10 Editorial. The shortened fluid fast and the Canadian Aneasthetists' Society's new guidelines for fasting in Aneasthetists' Society's new guidelines for fasting in 905-6.

11 Lister J. On anaesthetics, Part III. In: Homes' System of Surgery, Vol III, 3rd ed. London 1883 (Reprinted in: The Collected Papers of Joseph Lister. Birmingham: The Classics of Medicine Library, 1979: 171-2). 